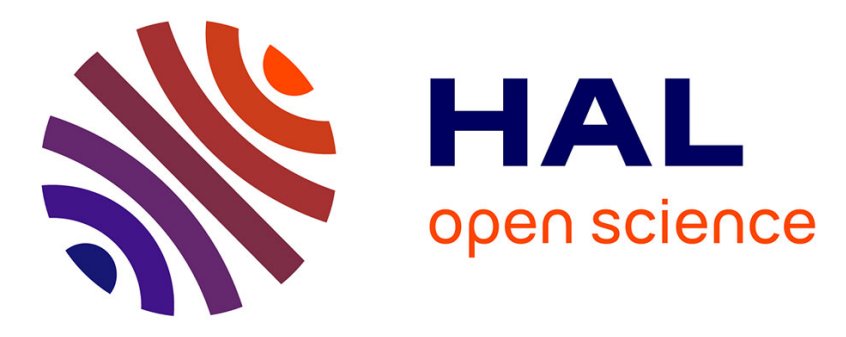

\title{
Expérimentation d'un modèle d'éducation thérapeutique pour patients polypathologiques dans les maisons de santé pluriprofessionnelles
}

\author{
Cyril Crozet, Nadia Boudraï-Mihoubi, Mariane Alphonse, François Frété, \\ Jean-François d'Ivernois
}

\section{To cite this version:}

Cyril Crozet, Nadia Boudraï-Mihoubi, Mariane Alphonse, François Frété, Jean-François d'Ivernois. Expérimentation d'un modèle d'éducation thérapeutique pour patients polypathologiques dans les maisons de santé pluriprofessionnelles. Éducation thérapeutique du patient / Therapeutic patient education , 2017, 9 (1), pp.10107. 10.1051/tpe/2017009 . hal-01685487

\section{HAL Id: hal-01685487 \\ https://sorbonne-paris-nord.hal.science/hal-01685487}

Submitted on 16 Jan 2018

HAL is a multi-disciplinary open access archive for the deposit and dissemination of scientific research documents, whether they are published or not. The documents may come from teaching and research institutions in France or abroad, or from public or private research centers.
L'archive ouverte pluridisciplinaire HAL, est destinée au dépôt et à la diffusion de documents scientifiques de niveau recherche, publiés ou non, émanant des établissements d'enseignement et de recherche français ou étrangers, des laboratoires publics ou privés. 


\title{
Expérimentation d'un modèle d'éducation thérapeutique pour patients polypathologiques dans les maisons de santé pluriprofessionnelles
}

\author{
Cyril Crozet ${ }^{1, *}$, Nadia Boudraï-Mihoubi ${ }^{1}$, Mariane Alphonse ${ }^{2}$, François Frété ${ }^{3}$ et Jean-François \\ d'Ivernois ${ }^{1}$ \\ ${ }^{1}$ Laboratoire Éducations et Pratiques de Santé (LEPS, EA 3412), Université Paris 13 -Sorbonne Paris Cité, Bobigny, France \\ 2 Service prévention santé Caisse Centrale de la Mutualité Sociale Agricole, Bagnolet, France \\ ${ }^{3}$ Caisse Centrale de la Mutualité Sociale Agricole, Bagnolet, France
}

Reçu le 30 juin 2017 / Accepté le 17 octobre 2017

\begin{abstract}
Résumé - Introduction: La forte prévalence des maladies chroniques s'accompagne d'une sousestimation des multimorbidités. Cette réalité se retrouve dans les soins de premier recours. Le programme d'éducation thérapeutique (ETP) polypathologie, d'après un modèle conçu par le laboratoire éducation et pratiques de santé (LEPS, EA 3412), a été proposé par la Mutualité sociale agricole (MSA) à des patients atteints de plusieurs maladies chroniques fréquentant des maisons de santé pluriprofessionnelles (MSP). Il a été expérimenté en 2015-2016. Objectif: Concevoir, valider et expérimenter un modèle opérationnel d'ETP polypathologie pour patients atteints de maladies cardiovasculaires, diabète, et/ou bronchopneumopathie chronique obstructive, éduqués dans deux MSP. Méthode : L'évaluation des patients par questionnaire (avant et immédiatement après éducation) a porté sur leurs connaissances des causes et des moyens de gestion de leurs maladies, sur leurs sentiments de confiance, de maitrise et d'efficacité. Les compétences acquises ont été interrogées six mois après l'ETP. Résultats : 47 patients ont été évalués (35 patients ont 2 pathologies, 11 en ont 3 et 1 en a 4). Après éducation, on constate un accroissement de leurs connaissances utiles : explication des maladies et de leur causes (T1 : 38,5\%; T2: 88,4\%, p $<0,001$ ), explication du but de chacun des médicaments (T1: $50 \%$; 2 : $100 \%, \mathrm{p}<0,001)$. Ils expriment leur confiance dans les traitements qu'ils prennent (T1: 84,1\%; T2: 95,4\%, p <0,05). Ils déclarent être capables de reconnaitre leurs propres crises (T1:46,1\%; T2:84,6\%, p <0,001), de savoir y faire face (T1 : $42,3 \% ; \mathrm{T} 2: 88,5 \%, \mathrm{p}<0,005)$ et de savoir sur qui ou quoi compter pour obtenir une aide (T1 : 84,6\%; T2 : $100 \%, \mathrm{p}<0,05)$. Parmi les compétences acquises pour la gestion quotidienne de leurs maladies, on note la pratique d'une activité physique régulière $(\mathrm{T} 1: 46,1 ; \mathrm{T} 2: 80,8 \%, \mathrm{p}<0,001)$, une adaptation de l'alimentation $(\mathrm{T} 1: 46,1 \% ; \mathrm{T} 2: 92,3 \%, \mathrm{p}<0,001)$, la capacité à gérer un traitement pluriel $(\mathrm{T} 1: 96,2 \%$; T2: $100 \%, p<0,01)$. Conclusion: La polypathologie constitue un défi pour l'ETP. Le modèle de programme d'ETP polypathologie que nous avons conçu et expérimenté semble pouvoir apporter une réponse pédagogique aux besoins de ces patients. D'autres recherches sont nécessaires pour confirmer l'efficacité et la robustesse du modèle.
\end{abstract}

Mots clés : éducation thérapeutique / programme expérimental / polypathologie / maisons de santé pluriprofessionnelles

\begin{abstract}
Experimentation of a model of therapeutic education for patients with multimorbidity in multi-professional health centers. Introduction: The high prevalence of chronic diseases is accompanied by an underestimation of multimorbidity. This reality is also found in primary care. The therapeutic patient education (TPE) program for multimorbidity, based on a model developed by the Education and Health Practice Laboratory (LEPS, EA 3412), has been proposed by the Mutualite Sociale Agricole (MSA), the french health insurance body for farmers, to patients with several chronic diseases attending multiprofessional primary care centers (MSPs). It was tested in 2015-2016. Objectives: To design, validate and experiment an operational model of TPE for multimorbidity patients with cardiovascular disease, diabetes,
\end{abstract}

$\overline{\text { *Auteur de correspondance }}$ : crozet@univ-paris13.fr 
and/or chronic obstructive pulmonary disease, educated in two MSPs. Method: Assessment of patients by questionnaire (before and immediately after education) focused on their knowledge of the causes and means for managing their illnesses, their feelings of trust, mastery and efficiency. Six months after the TPE program, patients were interviewed on the competencies they had acquired. Results: 47 patients were evaluated ( 35 patients had 2 pathologies, 11 had 3 and 1 had 4 ). After education, an increase in their useful knowledge has been observed: explanation of the diseases and their causes (T1: 38,5\%; T2: 88,4\%, $\mathrm{p}<0,001$ ), explanation of the effect of each drug (T1: 50\%; T2: 100\%, p < 0,001). They also expressed their confidence in the treatments they take (T1: 84,1\%; T2: 95,4\%, p <0,05). They declared themselves capable of recognizing their own crises (T1: 46,1\%; T2: 84,6\%, p <0,001), being able to face them (T1: $42,3 \%$; T2: $88,5 \%, \mathrm{p}<0,005)$ and knowing who to count on to get help (T1: 84,6\%; T2: 100\%, p < 0,05). Among the skills acquired for the day-to-day management of their diseases, we notice regular physical activity T1: 46,1; T2: 80,8\%, p <0,001), dietary adaptation (T1: $46,1 \%$; T2: $92,3 \%, p<0,001)$, ability to manage different treatments (T1: $96,2 \%$; T2: $100 \%, p<0,01)$. Conclusion: Multimorbidity is a challenge for TPE. The model of TPE program for multimorbidity that we designed and tested seems to provide an educational response to the needs of these patients. Further research is needed to confirm the effectiveness and strength of the model.

Key words: therapeutic patient education / experimental program / multimorbidity / multi-professional primary care centers

\section{Introduction}

L'allongement de l'espérance de vie en Occident s'accompagne d'une augmentation du nombre des malades chroniques dont la plupart sont affectés de multimorbidités $[1,2]$. Cette réalité concerne directement les soins de premier recours.

La recherche montre que la polypathologie conduit à la mort prématurée, occasionne des hospitalisations plus fréquentes, entraîne des dépressions et une mauvaise qualité de vie, se traduit par la prise de nombreux médicaments et une mauvaise observance générale, réduit le fonctionnement physique du patient [3].

Comme le souligne Tinetti [2], il semble que l'on n'ait pas encore pris pleinement conscience des changements d'organisation des soins rendus nécessaires par cette polypathologie. Au contraire, l'ensemble du système de soins est encore pensé en fonction de chaque maladie prise isolement. Se pose également le problème de la difficulté à définir clairement ce qui constitue la polypathologie.

Il est maintenant bien établi internationalement que l'ETP est indissociable de la prise en charge des maladies chroniques [4]. Mais la plupart des programmes d'ETP sont conçus en fonction d'une seule pathologie. Comme le soulignent d'Ivernois et Gagnayre [5]: «On sait, par exemple, comment éduquer un patient diabétique, un patient bronchitique, un patient hypertendu, mais on ne sait pas comment éduquer concrètement un patient atteint de ces trois maladies ». La difficulté consiste à repérer dans l'ensemble des apprentissages proposés au patient ce qu'il doit maîtriser pour assurer sa sécurité de façon prioritaire, mais aussi les priorités éducatives à privilégier en fonction des ses différentes pathologies et à réaliser l'organisation correspondante des séquences d'éducation [3]. De plus, l'accessibilité de l'ETP à tous les patients qui en auraient besoin dans le cadre de leur parcours de soins est problématique [3].

Il en découle la nécessité de faire bénéficier les patients polypathologiques une ETP adaptée [6]. Cependant, il n'existe pas actuellement de modèle didactique opératoire d'éducation thérapeutique du patient pour les personnes atteintes de plusieurs pathologies chroniques, ce que le laboratoire éducation et pratiques de santé (LEPS) de l'université Paris 13 souhaitait expérimenter et développer $[5,7,8]$. Ce modèle pourrait permettre de rationaliser l'organisation des différentes interventions d'ETP dans le parcours de soins d'un patient polypathologique, en particulier dans le cadre de structures de santé telles les maisons de santé pluriprofessionnelles (MSP) tournées à la fois vers la prévention et les soins curatifs [9], interventions renforcées par une coordination formalisée des soins. Les MSP représentent ainsi le siège idéal pour intégrer l'ETP de première ligne. C'est là que la "prescription» de l'ETP a le plus de chance d'être pertinente et que sa mise en œuvre est la plus susceptible de générer des résultats de santé meilleurs [9]. C'est la raison pour laquelle nous avons souhaité expérimenter ce modèle d'éducation thérapeutique dans ces structures.

Les objectifs de cette étude, réalisée par le LEPS dans le cadre d'un appel à projet de l'Institut de recherche en santé publique (IRESP) avec le soutien de la Mutualité sociale agricole (MSA) et en collaboration avec l'IPCEM (Institut en communication et éducation médicales), sont de concevoir, valider et expérimenter un modèle opérationnel d'ETP pour patients atteints de plusieurs pathologies chroniques, éduqués par des équipes de MSP.

Nous présentons ici successivement les étapes de la conception du modèle, de sa validation puis de son expérimentation pour des groupes de patients éduqués en MSP.

\section{Méthode}

\subsection{Choix de la population}

La coexistence de plusieurs pathologies dans la population d'adhérents de la MSA a été mesurée par cet organisme en 2012 [10]. On a pu constater, par exemple, que $43,8 \%$ des patients diabétiques classés en affection de longue durée (ALD) souffraient également d'une maladie cardiovasculaire, de même que $47,8 \%$ des patients atteints de bronchopneumopathie chronique obstructive (BPCO). 
C'est la raison pour laquelle il nous a paru pertinent d'envisager un modèle d'ETP polypathologie pour les patients atteints des pathologies suivantes:

- maladies cardiovasculaires (hypertension artérielle, insuffisance coronarienne, insuffisance cardiaque);

- diabète de type 2 ;

- bronchopneumopathie chronique obstructive.

\subsection{Modélisation de I'ETP pour patients polypathologiques}

Notre démarche initiale a consisté, après avoir documenté la réalité épidémiologique de la polypathologie en France à l'aide des enquêtes réalisées par la MSA [10], à réaliser une analyse de la littérature pour tenter d'obtenir une définition claire, opérationnelle du terme: "polypathologie» [7], base sémantique sur laquelle on pouvait s'appuyer. Nous avons ensuite mené une seconde analyse de la littérature permettant d'identifier à l'échelon international des expériences d'ETP pour patients polypathologiques [8].

Pour réaliser une modélisation d'un programme d'ETP polypathologie tenant compte des deux recherches précédentes, nous avons fait appel à des avis d'experts de la problématique à travers des méthodes telles que le focus group [11] et la méthode Delphi [12]. Deux focus groups ont été mis en œuvre (novembre 2013 à février 2014) réunissant 14 experts. Leurs propositions de modèle ont été examinées et validées par une méthode Delphi organisée en trois tours, de mars à juin 2014, réunissant un panel de 21 experts: huit praticiens exerçant en MSP, six chercheurs en ETP, trois représentants de patients et quatre experts-praticiens de l'ETP en région (Tab. 1). Ce sont leurs conclusions qui ont constitué la validation du modèle.

Le premier focus group (12/11/2013) a associé des chercheurs en pédagogie de la santé et en santé publique (3), des praticiens exerçant en MSP (8), un représentant de patients (1), des responsables régionaux et nationaux de la MSA (2), afin d'identifier les besoins éducatifs des patients chroniques polypathologiques, les compétences correspondantes à leur faire atteindre et les conditions de faisabilité d'une ETP polypathologie en MSP. Un second focus group (25/02/2014), réunissant les mêmes participants a eu pour but, à partir des résultats du premier, de dresser une «maquette conceptuelle» du modèle pédagogique de l'ETP polypathologie.

\subsection{Expérimentation du modèle pédagogique de l'ETP polypathologie}

Le modèle d'ETP pour patients polypathologiques a été expérimenté par des personnels volontaires de deux MSP (à Vicherey, Grand-Est et à Saint-James, Normandie) appartenant au réseau des MSP soutenues par la MSA. Ces professionnels de santé (3 infirmiers, 1 kinésithérapeute, 2 diététiciennes, 1 pédicure-spodologue, 1 psychologue clinicienne) avaient une expérience préalable d'éducateurs dans les programmes d'ETP itinérante pour patients atteints de pathologies cardiovasculaires [13]. Ils avaient tous participé aux focus groups.
Tableau 1. Participants au focus group et à la méthode Delphi. Table 1. Participants in the focus group and experts in the Delphi method group.

\begin{tabular}{lll}
\hline & $\begin{array}{l}\text { Focus } \\
\text { group }\end{array}$ & $\begin{array}{l}\text { Méthode } \\
\text { Delphi }\end{array}$ \\
\hline $\begin{array}{l}\text { Soignants éducateurs des maisons de santé } \\
\text { pluriprofessionnelles participants à l'expérience }\end{array}$ & 8 & 8 \\
$\begin{array}{l}\text { Patient affilié au régime de la MSA } \\
\text { Concepteurs du projet, membre du LEPS EA 3412 }\end{array}$ & 1 & 3 \\
$\begin{array}{l}\text { Membres du service de la prévention de la CCMSA } \\
\text { Chercheurs en ETP }\end{array}$ & 2 & 3 \\
Praticiens ETP et formateurs ETP & - & 3 \\
Total & - & 4 \\
& 14 & 21 \\
\hline
\end{tabular}

D'autre part, trois experts en méthodologie de l'ETP de l'Institut en communication et éducation médicales (IPCEM) [14] ont élaboré et/ou adapté des outils pédagogiques permettant d'accompagner les séquences de ce programme expérimental.

Une formation de trois jours en novembre 2014 a réuni les professionnels de santé des deux MSP et les experts de l'IPCEM. Cette formation a permis de simuler en temps réel le déroulement des séquences du modèle d'ETP polypathologie, de se familiariser avec l'emploi des outils pédagogiques de l'IPCEM et les outils d'évaluation conçus par l'équipe universitaire du LEPS.

L'expérimentation a consisté à éduquer à ce modèle, sur une durée d'un an à partir de mars 2015, un minimum de 30 patients par MSP (représentant aux moins trois cycles d'ETP polypathologie par an pour 3 groupes de patients), porteurs d'au moins deux pathologies dont l'association était reconnue prévalente par les enquêtes de la MSA. Ces trois groupes de patients ont tous suivi le même cycle d'ETP constitué d'un diagnostic éducatif, suivi de six séances de 1,5 heures ( 9 heures en tout) et conclu par une séance d'évaluation finale personnalisée.

\section{4 Évaluation}

Les objets de l'évaluation ont concerné :

- les effets observables chez les patients du programme expérimental d'ETP: acquisition de connaissances, de compétences d'auto-soins et d'adaptation et chez les patients, sentiment d'auto-efficacité perçue suite à l'éducation, compétences acquises à moyen terme ;

- le vécu des soignants éducateurs sur les aspects pédagogiques de l'expérimentation et son incidence sur leurs pratiques professionnelles.

\subsubsection{Les modalités d'évaluation}

Pour les patients, un pré-test (T1) de 14 questions préformées pour l'évaluation des connaissances (niveau de préoccupation et causes communes des maladies, rôles des médicaments, accidents/incidents, apport de l'activité physique et de l'alimentation, difficulté à suivre les moyens de 


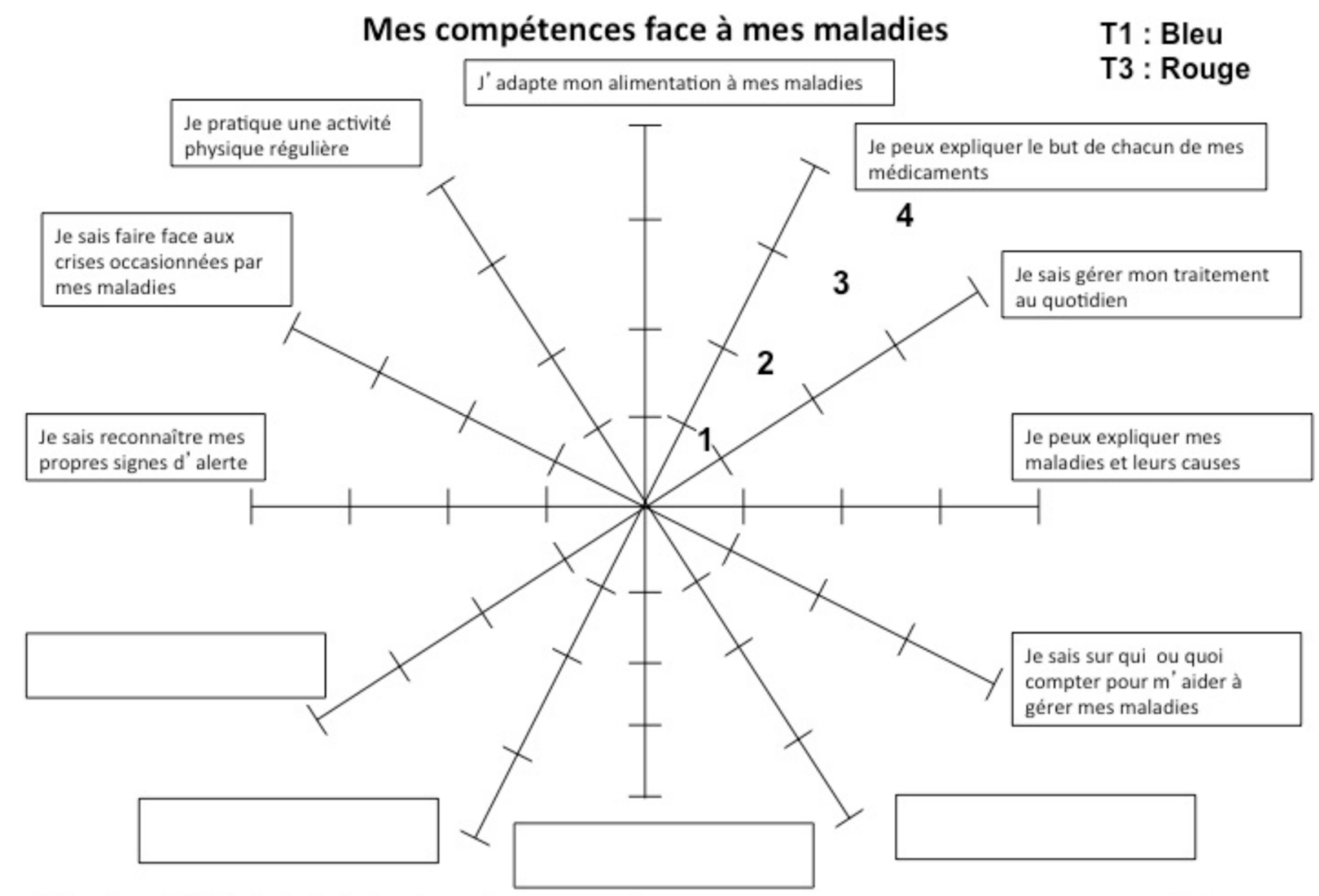

Npat. : __________ Légende : 1 : Pas du tout - 2 : Un peu - 3 : Assez - 4 : Tout à fait

Fig. 1. Rosace «mes compétences face à mes maladies», conception LEPS 2014.

Fig. 1. Rosace "my competencies in dealing with my illnesses". Conceived by LEPS, 2014.

traitement, aide à la gestion des maladies) complétées par trois questions avec échelle nuancée de réponses pour l'exploration du sentiment d'efficacité perçue, ainsi qu'une «rosace» à échelles multiple de Likert pour l'auto mesure des compétences (Fig. 1). Ce pré-test a été administré lors de la séance de diagnostic éducatif.

Le post-test (T2), administré lors de la séance d'évaluation finale personnalisée, juste après la dernière séance d'éducation, comportait les mêmes questions qu'au pré-test sauf la «rosace» qui leur a été proposée six mois après le cycle d'éducation. Ce post-test à moyen terme (T3) comportant la rosace d'auto-mesure des compétences a été envoyé aux patients par voie postale et/ou administré au fur et à mesure de leur fréquentation des MSP.

Pour les soignants éducateurs, un questionnaire de dix questions avec échelle nuancée de réponses et 11 questions ouvertes explorant leur vécu de l'expérience, auquel ils devaient répondre à la fin de trois cycles d'ETP qu'ils avaient conduits.

\subsubsection{Analyse des données}

Les réponses aux items de ces questionnaires ont fait l'objet d'une analyse statistique par le logiciel Biosta $\mathrm{TGV}^{\circledR}$; ils sont exprimés en nombres et pourcentages. Un Test $t$ de Student pour séries appariées a été utilisé pour mettre en évidence des différences significatives entre les résultats obtenus à $\mathrm{T} 1$ et $\mathrm{T} 2$ et à $\mathrm{T} 1$ et $\mathrm{T} 3$ (rosaces de compétences).

\section{Résultats}

\subsection{Modélisation de l'ETP polypathologique}

\subsubsection{Identification des besoins des patients et des compétences (premier focus group)}

Les participants au premier focus group ont souligné les besoins des patients polypathologiques concernant essentiellement la compréhension des maladies et des traitements (y compris la diététique), mais aussi leur difficulté à gérer quotidiennement plusieurs maladies. Ces besoins devraient pouvoir être identifiés lors d'un entretien individuel contextualisé à la vie du patient ou diagnostic éducatif [4].

De cette analyse des besoins est ressortie la formulation de compétences transversales: six compétences d'auto-soins et de huit compétences d'adaptation (ou compétences psychosociales) [15]. Dans le même temps ont été précisées les compétences d'auto-soins spécifiques à chaque maladie (Tab. 2).

\subsubsection{Conception du modèle d'ETP polypathologique}

L'existence de ces compétences transversales a conduit, lors d'un second focus group, à envisager que la formation du patient soit modulaire, consistant en un «module majeur polypathologie» qui permette leur acquisition. Il représente l'essentiel de la formation.

Des modules «mineurs » centrés sur les aspects spécifiques à chaque maladie ou «modules monopathologie» comportant 
Tableau 2. Compétences à acquérir par le patient polypathologique.

Table 2. Competencies that the patient with multimorbidity has to acquire.

\begin{tabular}{lll}
\hline Compétences transversales d'auto-soins (6) & Compétences transversales d'adaptation (8) & Compétences spécifiques à une pathologie \\
\hline S'expliquer ses maladies, les traitements & Informer, éduquer son entourage & Réaliser un geste technique \\
Identifier les facteurs de risques de ses & Exprimer ses besoins, solliciter l'aide de son & Reconnaître, interpréter une crise \\
maladies & entourage & \\
Appliquer la conduite à tenir en cas & Utiliser les ressources du système de soins, faire & \\
d'urgence, d'aggravation & valoir ses droits \\
Planifier, pratiquer la prise du traitement & Analyser les informations reçues sur ses maladies \\
(observance) & et ses traitements \\
Planifier, pratiquer une activité physique & Faire valoir ses choix de santé \\
Réaliser un équilibre diététique & Exprimer ses sentiments relatifs à la maladie et & \\
& mettre en œuvre des conduites d'ajustement & \\
& Établir des liens entre sa maladie et son histoire de vie \\
& Formuler un projet, le mettre en œuvre & \\
\hline
\end{tabular}

à la fois des objectifs théoriques et techniques viennent s'insérer dans le tronc commun du «module polypathologie ».

Cependant, les compétences de gestion des crises (anticipation, repérage, identification) constituent une priorité pédagogique. C'est la raison pour laquelle elles sont abordées en priorité dans les modules «monopathologie» correspondants.

\subsubsection{Organisation du modèle pédagogique}

Le modèle pédagogique est constitué de plusieurs séquences : une est consacrée au diagnostic éducatif préalable à la formation, une autre à six séances d'ETP et une, enfin, dédiée à l'évaluation et au réajustement personnalisé après la formation [16].

Le diagnostic éducatif polypathologie explore plusieurs dimensions cognitives et psychosociales:

- la connaissance, la compréhension des maladies, de leur accumulation et de leur intrication mutuelle et de la résultante en termes de réduction de la qualité de vie;

- la compréhension des différents traitements (y compris les contraintes diététiques qui peuvent s'accumuler), de leur potentialisation et des effets secondaires ou adverses, ainsi que la difficulté à leur gestion quotidienne ;

- la priorisation effectuée par le patient entre ses différentes maladies et le «rationnel-patient» qui sous-tend cette priorisation;

- le vécu de la polypathologie (image de soi, stigmatisation, atteinte de la vie affective, familiale et sociale).

Les six «séances» du programme Tab. 3) sont conçues pour «coller» aux besoins éducatifs de patients souffrant de polypathologies. Leurs titres ont été rédigés de façon à être compréhensibles par tous les patients:

- séance 1: ma maladie/mes maladies;

- séance 2 : mon traitement/mes traitements;

- séance 3 : alertes / gestion de crises / incidents / accidents ;

- séance 4 : en mouvement;

- séance 5 : qu'est-ce qu'on mange et comment?

- séance 6: je m'aide/tu m'aides/ils m'aident.

L'évaluation pédagogique du modèle est constituée d'une évaluation formative lors du diagnostic éducatif, à la fin de chaque séance et même dans chaque module pluri et monopathologique. Une évaluation finale personnalisée est proposée aux patients juste après la $6^{\mathrm{e}}$ séance.

\subsubsection{Pédagogie du modèle}

Le fonctionnement d'un tel modèle associant des activités éducatives communes et d'autres spécifiques pour un même groupe de patients nécessite de convoquer des modèles pédagogiques opératoires. Les théories et les expériences de pédagogie différenciée $[17,18]$ nous ont aidés à tenir compte de la diversité culturelle des patients, de leur capacité d'apprentissage, de la nécessité de faire porter leur attention tour à tour sur ce qui est transversal à toutes les maladies et spécifique à chacune d'entres elles, de leur apprendre à prioriser de façon cohérente leurs différentes maladies. L'apport du socioconstructivisme $[19,20]$ nous a aidés pour la planification de l'apprentissage des patients par compétences (auto-soins et d'adaptation) en les déclinant secondairement en objectifs éducatifs opératoires et en activités pédagogiques mobilisées dans les modules transversaux et spécifiques.

\subsubsection{Validation du modèle par la méthode Delphi}

Le premier texte (issu du second focus group) et les suivants ont été envoyés par mail aux experts du panel Delphi. Il leur a été demandé d'exprimer leurs jugements et propositions dans la partie «commentaires» mise à leur disposition. Les différents tours ont généré un certain nombre de changements et de commentaires concernant en particulier la reformulation du contenu des séances d'ETP, la place respective des compétences d'auto-soins et d'adaptations selon les séances, l'apport et l'emploi des outils pédagogiques.

Le modèle, tel que validé par le panel Delphi, est présenté dans le tableau 3.

\section{2 Évaluation du programme expérimental}

\subsection{1 Évaluation pédagogique des patients éduqués}

Le nombre de patients évalués au pré-test (T1) est de 47, ainsi qu'au au post-test (T2). Il se réduit à 27 au post-test à moyen terme (T3). L'âge moyen des patients est de 66,5 ans $(\min =53, \max =78)$ et le sex ratio de 0,7 . 


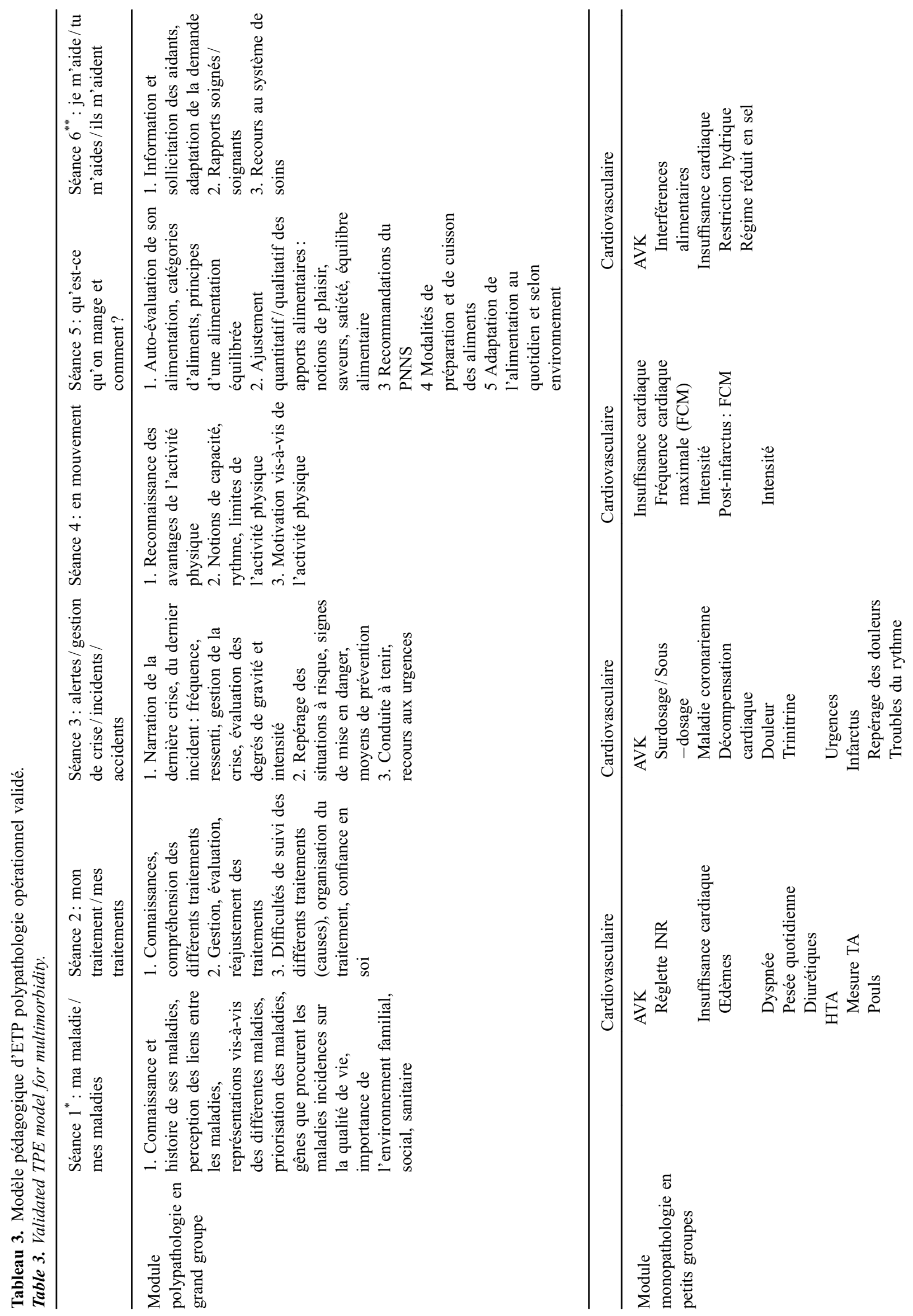

Page 6 de 11 
Tous les patients sont polypathologiques. Les deux affections les plus fréquentes sont l'hypertension artérielle (HTA) et le diabète de type $2: 43$ patients ont une hypertension artérielle, 37 patients sont diabétiques, 16 patients ont une maladie coronarienne, neuf patients souffrent d'insuffisance cardiaque (IC), deux patients ont une bronchopneumopathie chronique obstructive (BPCO).

Concernant l'association des pathologies, 35 patients ont deux pathologies, 11 patients ont trois pathologies, un patient a quatre pathologies.

\subsubsection{Niveau de préoccupation des patients vis-à-vis de leurs différentes pathologies}

Au pré-test, 22 patients déclarent qu'ils se préoccupent le plus du diabète $(46,8 \%)$ puis, dans l'ordre, de l'hypertension artérielle (HTA): dix patients $(21,3 \%)$, de la maladie coronarienne (MC) : quatre patients $(8,5 \%)$, de l'insuffisance cardiaque (IC) : trois patients $(6,4 \%)$ et de la bronchopneumopathie chronique obstructive BPCO : un patient.

$\mathrm{Au}$ post-test, on observe une augmentation de la préoccupation exprimée par les patients vis-à-vis de leurs maladies. Le diabète préoccupe désormais 29 patients $(61,7 \%)$, l'HTA, 15 patients, (31,9\%), l'IC (6 patients, $12,8 \%)$. L'attention portée à la maladie coronarienne reste stable (4 patients, $8,5 \%$ ).

On constate donc qu'après éducation, davantage de patients diabétiques $(+15)$, de patients hypertendus $(+5)$ et de patients insuffisants cardiaques $(+3)$ se préoccupent de l'importance de leurs maladies, ce qui pourrait s'interpréter comme la capacité à mieux savoir les reconnaître comme telles et les prioriser les unes par rapport aux autres.

\subsubsection{Connaissance des causes communes aux différentes pathologies}

Nous avons exploré les connaissances des patients sur les causes communes à leurs différentes maladies avant et après éducation. On constate quelques changements entre le pré-test et le post-test dans l'ordre des causes communes évoquées (le surpoids $[+12,8 \%]$, le stress $[+14,9 \%]$, l'alimentation $[+10,6 \%] \ldots)$. Par contre, le nombre de réponses augmente globalement au post-test (en \% dans les parenthèses). Il pourrait donc s'agir d'un gain de connaissances lié à l'éducation.

\subsubsection{Connaissance du rôle des médicaments}

Après éducation, les patients améliorent globalement leurs connaissances sur les médicaments qu'ils prennent pour traiter leurs maladies. Nous avons considéré essentiellement la progression de ces connaissances chez les patients concernés par une catégorie de médicaments (par exemple, la connaissance des médicaments antihypertenseurs chez les patients souffrant d'HTA). On observe au total que les connaissances des patients sur les médicaments sont restées stables vis-à-vis des antidiabétiques, des anti-vitamine $\mathrm{K}$, des statines, des antibiotiques. Cela signifie que les patients diabétiques avaient des connaissances initiales déjà élevées sur les hypoglycémiants oraux $(86,5 \%)$, que les patients bronchitiques connaissaient déjà bien le rôle des corticoïdes inhalés 
$(100 \%)$ et des antibiotiques (100\%), que tous les patients coronariens $(100 \%)$ comprenaient le rôle des statines.

Par contre, les patients ont réalisé, grâce à l'éducation, un apprentissage sur les antihypertenseurs (T1: 83,7\%; T2: $100 \%$ ), les bétabloquants (T1 : 41,4\%; T2: 63,4\%). Ces connaissances ont été plus élevées encore (et plus particulièrement chez les patients hypertendus) en ce qui concerne les inhibiteurs de l'enzyme de conversion/antagonistes des récepteurs de l'Angiotensine 2 (T1 : 23,2\%; T2: 40,81\%), les diurétiques $\mathrm{T} 1: 31,1 \% ; \mathrm{T} 2: 53,3 \%)$, les digitaliques (T1 : $10 \% ; \mathrm{T} 2: 30 \%$ ), les dérivés nitrés ( $\mathrm{T} 1: 13,3 \% ; \mathrm{T} 2: 26,6 \%)$ et l'aspirine (T1: $60 \%$; T2: 93,3\%).

\subsubsection{Connaissance des incidents et accidents susceptibles d'être rencontrés}

Nous avons cherché à apprécier l'évolution des connaissances des patients vis-à-vis des incidents et accidents qu'ils sont susceptibles de rencontrer. Nous constatons que les connaissances des patients se sont améliorées entre T1 et T2 vis-à-vis de tous les types d'accidents : AVC (T1 : 66\%; T2 : 76,6\%), hypoglycémie (T1 : 51,1\%, T2: 63,8\%), infarctus du myocarde (T1: 42,6\%; T2: 61,7\%), hyperglycémie (T1: $29,8 \% ; \mathrm{T} 2: 51,1 \%$ ), hémorragie (T1 : $10,6 \% ; \mathrm{T} 2: 17 \%)$ infection pulmonaire (T1: 8,5\%; T2: 12,8\%).

\subsubsection{Connaissances sur l'activité physique et l'alimentation}

$\mathrm{Au}$ post-test $\mathrm{T} 2$, les patients sont plus nombreux à reconnaître l'activité physique comme bénéfique pour la gestion de leurs maladies (T1 : 77\%; T2 : 100\%).

Cet accroissement des connaissances s'observe également en ce qui concerne l'alimentation. Les patients déclarent être désormais plus vigilants :

- vis-à-vis des sucres ( $\mathrm{T} 1:$ 95,3\%; $\mathrm{T} 2: 98,5 \%$ ), en particulier, les patients souffrant d'HTA, d'IC et de MC, les patients diabétiques ayant déjà atteint ce niveau de connaissance ;

- vis-à-vis des graisses chez tous les patients (T1 : 89,2\% ; T2: $96 \%$ );

- vis-à-vis du sel chez tous les patients (T1: 78,4\%; T2 : $97,3 \%$ ).

\subsubsection{Difficultés à suivre les traitements}

Suite à l'éducation, les patients déclarent que certains moyens de traitement leur semblent relativement moins difficiles à suivre. C'est le cas de l'alimentation (T1: 53,2\%; T2: 46,8\%), de l'activité physique (T1: 46,8\%; $\mathrm{T} 2: 44,7 \%)$ et des médicaments (T1 : 10,6\%; T2 : 2,1\%) Par contre, pour trois patients fumeurs l'éducation ne semble pas avoir modifié leur opinion vis-à-vis de l'arrêt du tabac qui leur parait toujours aussi difficile.

\subsubsection{Aide aux patients dans la gestion de leurs maladies}

À la question de savoir sur qui ou sur quoi les patients peuvent compter pour se faire aider dans la gestion de leur maladie, les réponses indiquent une progression des avis positifs vis-à-vis :
- du pharmacien (T1: 19, 2\%; T2: 59,6\%);

- des maisons de santé pluriprofessionelles (T1: 38,3\%; $\mathrm{T} 2: 51,1 \%$ )

- des autres soignants (T1:36,2\%; T2: 48, 9\%);

- des médecins spécialistes (T1 : 34\%; T2: 46,8\%);

- des associations de patients (T1:2,1\%; T2: 14,9\%).

D'autre part, les patients considèrent encore au post-test qu'ils doivent compter avant tout sur eux-mêmes $(87,2 \%)$, sur leur médecin généraliste $(80,9 \%)$ et sur leur famille $(68,1 \%)$.

\subsubsection{Sentiments de confiance, de maîtrise et d'efficacité}

Immédiatement après l'éducation, les patients $(n=44)$ sont interrogés sur leurs sentiments de confiance, de maîtrise et d'efficacité immédiate au $\mathrm{T} 1$ et $\mathrm{T} 2$. Ils déclarent avoir confiance dans les traitements qu'ils prennent (T1: 84,1\%; $\mathrm{T} 2: 95,4 \%, \mathrm{p}<0,05)$ et qu'ils se sentent capables de faire face aux crises (T1 : 52,3\%; T2: 79,5\%, p <0,001). Par contre, leur sentiment de capacité à gérer leurs maladies demeure stable (T1: 81,8\%; T2: 84,1\%). Ceci contraste avec les compétences qu'ils déclarent avoir acquises six mois après l'éducation.

\subsubsection{Compétences acquises à six mois}

Par rapport au pré-test, les compétences que les patients $(n=26)$ estiment avoir acquises six mois après l'ETP (T3) concernent :

- des connaissances utiles : explication des maladies et leurs causes (T1 : 38,5\%; T3: 88,4\%, p <0,001), explication du but de chacun des médicaments (T1: $50 \%$; T3 : $100 \%$, $\mathrm{p}<0,001)$;

- des capacités mobilisables en cas de crises : reconnaître ses propres crises (T1 : 46,1\%; T3: 84,6\%, p <0,001), faire face aux crises occasionnelles (T1 : 42,3\%; T3 : 88,5\%, $\mathrm{p}<0,005)$, savoir sur qui ou sur quoi compter pour être aidé (T1: 84,6\%; T3 : $100 \%, \mathrm{p}<0,05)$;

- des comportements de santé au quotidien: pratiquer une activité physique régulière (T1: 46,1; T3: 80,8\%, $\mathrm{p}<0,001)$, adapter son alimentation (T1: 46,1\%; T3: $92,3 \%, \mathrm{p}<0,001)$, savoir gérer son traitement (T1: $96,2 \% ; \mathrm{T} 3: 100 \%, \mathrm{p}<0,01)$.

\subsection{2 Évaluation par les soignants-éducateurs}

Neuf soignants-éducateurs issus de deux MSP ont été formés à la méthodologie du modèle. Sept d'entre eux ont répondu au questionnaire explorant leur vécu de l'expérience après avoir animé trois cycles du programme d'ETP polypathologie. Leurs réponses ont permis de mettre en évidence différents freins à l'application de ce modèle expérimental; ils concernent le recrutement des patients qui a été «plutôt difficile», la communication avec les médecins traitants, réticents à leur envoyer des patients pour participer au programme d'ETP. Sa densité a pu aussi constituer un obstacle. Les répondants ont souhaité, en majorité, disposer d'un peu plus de temps pour mener à bien les séances d'ETP : plutôt que de multiplier le nombre de séances, ils suggèrent 
d'augmenter un peu leur durée. Ils ont souligné d'autre part que malgré la formation qu'ils avaient reçue, un temps d'appropriation des séances et des outils pédagogiques leur avait été nécessaire.

Ils ont, par ailleurs, exprimé leur satisfaction vis-à-vis des outils pédagogiques conçus par l'IPCEM qui leur ont paru pratiques, ludiques et faciles d'utilisation. La structure des séquences d'ETP leur a semblée "parfaitement adaptée». Ils ont précisé que l'animation en binôme avait été «importante» pour gérer les temps de formation aux compétences transversales et spécifiques. Enfin, les patients n'ont pas, selon eux, manifesté de réticence vis-à-vis des ateliers pratiques «monopathologie ». Parfois même, des patients non concernés par ces ateliers y ont participé.

\section{Discussion}

La littérature internationale n'est pas très riche en expériences d'ETP polypathologie, ce qui indique qu'il s'agit d'un secteur de recherche encore peu exploré.

Ainsi, une revue systématique menée par Deccache et Albano en 2014 pour la décennie 2004-2013 n'a retrouvé que neuf articles spécifiquement consacrés à ce sujet [8] tandis que Smith et al. en 2016 ont trouvé 18 articles sur des interventions complexes pour des patients polypathologiques [21]. La majorité de ces articles étaient des études randomisées contrôlées qui n'ont apporté que des bénéfices, limités essentiellement à l'activité physique et à l'alimentation. Bien que les stratégies pédagogiques décrites par les auteurs aient eu recours à différents formats (éducation de groupe, éducation en face à face, simulation, Web, carte mentale), une seule étude [22] faisait référence à un modèle pédagogique, celui du CDSMP (Chronic Disease Self Management Program) de K. Lorig [23], mais sans pourtant convaincre qu'il soit totalement adapté à la polypathologie. Le besoin d'un modèle opératoire, tel que celui que nous proposons, est donc d'actualité.

Deux auteurs $[24,25]$ ont mis l'accent comme nous l'avons fait sur la nécessité d'apprendre aux patients à établir une priorité entre leurs différentes maladies. Dans notre étude, nous avons pu constater que cet objectif a été atteint puisque après éducation, les patients savent réorienter leur niveau de préoccupation vis-à-vis de pathologies prioritaires.

Certains auteurs, Harrison [22], Lenferink [25], O’Toole [26] et Eakin [27] ont cherché, comme dans notre modèle, à développer chez les patients, des compétences transversales en ce qui concerne le traitement médicamenteux, l'activité physique et l'alimentation. Ces compétences, recommandées par plusieurs autres auteurs, d'Ivernois [5], Lorig [23,28] semblent faire l'objet d'un consensus en ce qui concerne l'ETP pour patients polypathologiques.

Les résultats que nous obtenons s'inscrivent dans cette ligne puisque suite à l'ETP, les patients déclarent que ces moyens de traitement leur semblent plus faciles à suivre. Cela n'aurait sans doute pas été possible sans un accroissement notable chez ces patients des connaissances correspondantes, y compris sur le rôle des médicaments et les situations accidentelles.

D'autres compétences essentielles semblent acquises chez les patients des deux MSP de notre expérience: savoir faire face aux situations d'urgence, et savoir par qui ou quoi se faire aider. Au total, les sentiments de confiance en leurs propres compétences et leur efficacité personnelle sont augmentés après éducation chez les patients polypathologiques, ce qui pourrait constituer un indicateur synthétique de l'efficacité de ce programme.

Pour les soignants-éducateurs, la difficulté à recruter des patients a constitué un obstacle mais qui ne semble pas spécifiquement lié à l'ETP polypathologie. Ils ont été globalement satisfaits de l'application du modèle, des objectifs et contenus des séquences et de la formation pédagogique qu'ils ont reçue. Il sera important dans l'avenir de bien calibrer la durée totale du cycle d'ETP et de ses séquences, compte tenu des besoins exprimés par le patient lors du diagnostic éducatif polypathologie dont l'importance est ici plus que jamais essentielle. Comme nous l'avons vérifié, Il ne semble pas possible de lancer des soignantséducateurs dans l'organisation et l'exécution de programme d'ETP polypathologie sans leur avoir dispensé au préalable une formation pédagogique adéquate. Cette formation constituerait une spécialisation de la formation réglementaire initiale dite des $« 40 \mathrm{~h} »$.

\subsection{Limites}

Cette étude comporte plusieurs limites. Le nombre de participants (14) aux deux focus group peut paraître réduit. Il correspond cependant aux exigences de la littérature [11]. Bien que trois patients aient été inclus dans la méthode Delphi et un patient dans les focus group, nous n'avons pas réalisé d'enquête préalable auprès de patients polypathologiques. La participation des experts a été satisfaisante avec une érosion certaine au troisième tour du Delphi. Cette méthode demeure essentiellement qualitative. À ce titre, le «consensus » n'a aucune prétention de représentativité car les résultats de l'enquête Delphi reposent uniquement sur l'expertise des participants » [12].

Dans l'expérimentation du modèle, on peut regretter que le nombre de soignants-éducateurs impliqués soit aussi limité, de même que celui des patients éduqués. Bien que les deux MSP se situent dans des régions éloignées du territoire national et que les résultats à l'évaluation soient concordants, nous sommes cependant conscients de la difficulté à les généraliser. D'autres études impliquant un plus grand nombre de MSP s'avèrent nécessaires pour conclure à l'efficience pédagogique du modèle

\section{Conclusion}

Le modèle pédagogique d'ETP polypathologie que nous proposons est opératoire pour les personnes atteintes de plusieurs pathologies chroniques. Dans la conception du modèle, nous avons eu le souci d'intégrer tous les acteurs de l'ETP en les sollicitant à chaque étape de la recherche, du focus group à la méthode Delphi. La diversité des experts sollicités nous a aidés à concevoir un dispositif participatif nécessitant des outils éducatifs adaptés et des compétences pédagogiques particulières.

Ce modèle pédagogique d'ETP polypathologie met l'accent sur l'intelligibilité des patients vis-à-vis de leurs maladies et de leurs traitements, sur le rôle transversal qu'ils accordent à la diététique et à l'activité physique. Les résultats positifs de l'évaluation expérimentale auprès de 47 patients 
nous confortent sur les possibilités pour les patients polypathologiques de maîtriser les compétences indispensables à la gestion plurielle de leurs problèmes de santé et d'exprimer leurs choix éclairés.

Nous pensons que la mise en œuvre d'un tel modèle pédagogique d'éducation thérapeutique polypathologie produira des changements des pratiques des professionnels, en particulier dans les maisons de santé pluriprofessionelles, afin de répondre à la spécificité de l'éducation thérapeutique polypathologie par rapport à des programmes centrés sur une seule maladie.

Remerciements. Les auteurs remercient les soignants-éducateurs du pôle de santé de Saint-James (D. Beaucé C. Camous C. Dufosse, C. Lhopital,) et de la maison de santé pluriprofessionnelle de Vicherey (S. Audinot, A. Cher, P. Choquert), ainsi que tous les patients qui ont participé à l'expérimentation du programme d'ETP polypathologie.

Nous remercions également le Dr T. Ancelle pour ses conseils en statistique et la Pr MG. Albano pour son aide à la traduction.

Cette recherche a bénéficié de l'aide conjointe de la DGS, de l'INCA et de la MSA dans le cadre de l'appel à projet lancé par l'IReSP en 2012.

Conflits d'intérêts. Les auteurs déclarent n'avoir aucun lien d'intérêt concernant les données publiées dans cet article.

\section{Liste des abréviations}

$\begin{array}{ll}\text { ALD } & \text { affections de longue durée } \\ \text { ETP } & \text { éducation thérapeutique du patient } \\ \text { IPCEM } & \text { Institut en communication et éducation médicales } \\ \text { IRESP } & \text { Institut de recherche en santé publique } \\ \text { LEPS } & \text { laboratoire éducations et pratiques de santé } \\ \text { MSA } & \text { Mutualité sociale agricole } \\ \text { MSP } & \text { maison de santé pluriprofessionnelle }\end{array}$

\section{Références}

1. Barnett K, Mercer SW, Norbury M, Watt G, Wyke S, Guthrie B. Epidemiology of multimorbidity and implications for health care, research, and medical education: a cross-sectional study. Lancet 2012; 380:37-43.

2. Tinetti ME. Designing health care for the most common chronic condition-multimorbidity. JAMA 2012; 307:2493-2494.

3. Smith SM, Soubhi H, Fortin M, Hudon C, O'Dowd T. Managing patients with multimorbidity: systematic review of interventions in primary care and community settings. BMJ 2012; 345:e5205.

4. d'Ivernois JF, Gagnayre R. Apprendre à éduquer le patient. $5^{\mathrm{e}}$ éd. Paris: Maloine; 2016.

5. d'Ivernois JF, Gagnayre R. Éducation thérapeutique chez les patients pluripathologiques, propositions pour la conception de nouveaux programmes d'ETP. Educ Ther Patient/Ther Patient Educ 2012; 5:201-204.

6. Bratzke L, Muehrer RJ, Kehl KA, Lee KS, Ward EC, Kwekkeboom KL. Self-management priority setting and decision-making in adults with multimorbidity: a narrative review of literature. Int J Nurs Stud 2015; 52(3):744-755.

7. Lefèvre T, d'Ivernois JF, De Andrade V, Crozet C, Lombrail P, Gagnayre R. What do we mean by multimorbidity? An analysis of the literature on multimorbidity measures, associated factors, and impact on health services organization. Revue d'Épidémiologie et de Santé Publique 2014; 62:305-314.

8. Deccache C, Albano MG, de Andrade V, Crozet C, d'Ivernois JF. Therapeutic patient education for patients with multimorbidity: a recent literature review. Educ Ther Patient/Ther Patient Educ 2014; 6(2):2015.

9. Fournier C. Les maisons de santé pluriprofessionnelles, une opportunité pour transformer les pratiques de soins de premier recours: place et rôle des pratiques préventives et éducatives dans des organisations innovantes. Santé publique et épidémiologie. Université Paris Sud-Paris XI, 2015. Français. $<$ NNT: 2015PA11T011>. <tel-01149605>.

10. Direction des études des répertoires et des statistiques de la Mutualité sociale agricole. Multimorbidités chroniques des affections de longue durée au régime agricole au 31 décembre 2012 et lien avec l'éducation thérapeutique du patient. Étude. MSA Mai 2015, 30 p.

11. Duchesne S, Haegel F. L'enquête et ses méthodes: l'entretien collectif. Paris: Armand Colin; 2013.

12. Booto Ekionea JP, Prosper B, Plaisent M. Consensus par la méthode Delphi sur les concepts clés des capacités organisationnelles spécifiques de la gestion des connaissances. Recherches qualitatives 2011; 29(3):168-192.

13. Crozet C, Van Bockstael V, d'Ivernois JF. Éducation thérapeutique de patients âgés cardio-vasculaires: le programme de la Mutualité sociale agricole. In: J. Faucoud, J. Bury, M. BalcouDebussche (coord.) Modèles, pratiques et évaluations de démarche d'éducation thérapeutique du patient. Paris: Éditions INPES, 2010, pp. 146-151.

14. IPCEM Association pour le développement de l'éducation thérapeutique (page d'accueil internet), France, (cité le 30 mai 2017). Disponible sur www.ipcem.org.

15. d'Ivernois JF, Gagnayre R, les membres du groupe de travail de l'IPCEM. Compétences d'adaptation à la maladie du patient: une proposition. Educ Ther Patient/Ther Patient Educ 2011; 3(2): S201-S205.

16. HAS. Auto-évaluation annuelle d'un programme d'éducation thérapeutique du patient. Guide pour les coordonnateurs et les équipes. HAS, 2012.

17. de Lagaranderie A. Les profils pédagogiques. Paris: Bayard Édition; 1980.

18. Meirieu Ph. L'école mode d'emploi. Paris: ESF Editeur; 1995.

19. Jonnaert P. Compétences et socioconstructivisme: un cadre théorique. Bruxelles: De Boeck; 2009.

20. Wittorski R. Analyse du travail et production de compétences collectives. Paris: L'Harmattan 1997.

21. Smith SM, Wallace E, O'Dowd T, Fortin M. Interventions for improving outcomes in patients with multimorbidity in primary care and community settings. Cochrane Database of Systematic Reviews 2016, Issue 3. Art. No.: CD006560. DOI: 10.1002/ 14651858.CD006560.pub3.

22. Harrison M, Reeves D, Harkness E, Valderas J, Kennedy A, Rogers A, et al. A secondary analysis of the moderating effects of depression and multimorbidity on the effectiveness of a chronic disease selfmanagement programme. Patient Educ Couns 2012; 87:67-73.

23. Lorig KR, Sobel DS, Stewart AL, Brown BW Jr, Bandura A, Ritter $\mathrm{P}$, et al. Evidence suggesting that a chronic disease self management program can improve health status while 
reducing hospitalization: a randomized trial. Med Care 1999; 37: 5-14.

24. Bogner HR, de Vries HF. Integration of depression and hypertension treatment: a pilot, randomized controlled trial. Ann Fam Med 2008; 6:295-301.

25. Lenferink A, Frith P, van der Valk P, Buckman J, Sladek R, Cafarella $\mathrm{P}$, et al. A self-management approach using self initiated action plans for symptoms with ongoing nurse support in patients with chronic obstructive pulmonary disease (COPD) and comorbidities: the COPE-III study protocol. Contemp Clin Trials $2013 ; 36: 81-89$.
26. O' Toole L, Connolly D, Smith S. Impact of an occupation-based self-management programme on chronic disease management. Aust Occup Ther J 2013; 60:30-38.

27. Eakin EG, Bull SS, Riley KM, Reeves MM, McLaughlin P, Gutierrez S. Resources for health: a primary-care-based diet and physical activity intervention targeting urban Latinos with multiple chronic conditions. Health Psychol 2007; 26: 392-400.

28. Lorig KR, Ritter P, Stewart AL, Sobel DS, Brown BW, Bandura A, et al. Chronic disease self-management program: 2 years health status and health care utilization outcomes. Med Care 2001; 39(11):1217-1223.

Citation de l'article : Crozet C, Boudraï-Mihoubi N, Alphonse M, Frété F, d'Ivernois J-F. Expérimentation d'un modèle d'éducation thérapeutique pour patients polypathologiques dans les maisons de santé pluriprofessionnelles. Educ Ther Patient/Ther Patient Educ 2017; 9:10107. 